\title{
Risk factors for atypical lymph node metastasis in gastric cancer
}

\author{
Jinsol Jung', Sang II Yoon', Kang-Haeng Lee', Yongjoon Won'1, Sa-Hong Min'1, Young-Suk Park', Sang Hoon Ahn², \\ Do Joong Park ${ }^{2,3}$, Hyung-Ho Kim ${ }^{1,2}$ \\ 'Department of Surgery, Seoul National University Bundang Hospital, Seoul National University College of Medicine, Seongnam; \\ ${ }^{2}$ Department of Surgery, Seoul National University College of Medicine, Seoul; \\ ${ }^{3}$ Department of Surgery, Seoul National University Hospital, Seoul, Korea
}

Purpose: The present study aimed to evaluate atypical lymph node metastasis rates according to tumor depth, size, and location in patients with gastric cancer.

Methods: A total of 727 gastric adenocarcinoma patients, with metastasis to 1 or 2 lymph nodes, who underwent radical gastrectomy with curative intent from May 2003 to May 2017, were enrolled in this study. The characteristics of atypical (skip or transversal) metastases were evaluated according to the following risk factors: longitudinal versus circumferential location, size, and T stage of the tumor.

Results: The rates of skip and transversal metastases were $8.4 \%$ and $15.5 \%$, respectively. Skip metastases were present throughout, regardless of the primary tumor location. On the contrary, transversal metastases of gastric cancer were most frequently observed in the lower third region $(19.5 \%, P=0.002)$. When the size of the tumor is large $(>4 \mathrm{~cm})$, transversal metastasis was found to be significantly more common $(P=0.035)$, compared with skip metastasis, which was less common $(P=0.011)$. There was no significant correlation between atypical metastases and tumor depth.

Conclusion: Lower and larger tumors were more likely to have transversal metastases compared with others; however, skip metastases were less common in large tumors.

Keywords: Stomach neoplasms, Lymph node dissection

\section{INTRODUCTION}

Lymph node (LN) metastasis is very important for the prognosis of gastric cancer, and topics surrounding the amount of LN dissection during surgery remain controversial. Nodal metastasis of gastric cancer cells is relatively more aggressive than other cancers and removal is necessary even for micrometastasis nodes [1].

Received: Sep 18, 2019 Revised: Dec 11, 2019 Accepted: Dec 17, 2019 Correspondence to: Do Joong Park

Division of Gastrointestinal Surgery, Department of Surgery, Seoul

National University Hospital, Seoul National University College of

Medicine, 101 Daehak-ro, Jongno-gu, Seoul 03080, Korea

Tel: +82-31-787-7097, Fax: +82-31-787-4078

E-mail: djparkmd@snu.ac.kr

ORCID: Jinsol Jung (https://orcid.org/0000-0002-9458-8057), Do Joong Park (https://orcid.org/0000-0001-9644-6127)

Copyright (C) 2019 Korean Society of Surgical Oncology

This is an Open Access article distributed under the terms of the Creative Commons Attribution Non-Commercial License (http://creativecommons.org/licenses/by-nc/4.0) which permits unrestricted non-commercial use, distribution, and reproduction in any medium, provided the original work is properly cited.
Currently, D2 dissection is accepted as the gold standard of gastric cancer treatment. However, D1 dissection is also performed in early gastric cancer (EGC) without LN metastasis in Korea and Japan [2,3]. Endoscopic mucosal resection, endoscopic submucosal dissection, and sentinel node navigation surgery (SNNS) have been attempted to preserve gastric function and improve quality of life according to the recent minimal invasive tendency [4-8].

Gastric cancer has a relatively complex multidirectional lymphatic flow, and the rate of skip metastasis is not low, making it difficult to apply SNNS in the treatment of gastric cancer [9-11]. Therefore, if risk factors related to the possibility of atypical LN metastasis, such as skip metastasis, are identified, it is helpful to set an indication of minimally invasive surgery.

To date, skip metastases have been studied several times; however, to the best of our knowledge, studies on transversal metastases are rare $[12,13]$. There were also only a few studies on atypical LN metastasis in advanced gastric cancer (AGC). The purpose of this study was to determine the distribution of atypical LN metastasis according to tumor location and depth. 


\section{METHODS}

This study was approved by Institutional Review Board of the Seoul National University Bundang Hospital (IRB No: B-2001/586-110). Consecutive gastric tissue specimens were obtained retrospectively from 6,827 patients who had undergone curative resection for gastric cancer at Seoul National University Bundang Hospital (Seongnam, Korea) between May 2003 and May 2017. Palliative resection, R1 or R2 resection, and multiple lesions were excluded from the study; only tumors in stage $\mathrm{pN} 1$ were selected. Therefore, a total of 727 patients were selected for this study (Fig. 1).

Medical charts and pathology reports were reviewed to obtain clinical and pathologic data, including age, sex, pathological TNM staging according to the American Joint Committee on Cancer 7th edition, depth of invasion, angiolymphatic invasion, neural invasion, and histologic subtype.

Skip metastases and transversal metastases were considered as types of atypical LN metastases. Skip metastasis was defined as the presence of a metastatic LN in an extraperigastric area without perigastric involvement. Perigastric LNs were LNs numbered 1 to 6. Extraperigastric LNs were the remaining ones. Transversal metastasis was defined as metastasis to the opposite side of the tumor location, without metastasis to the tumor side.

Statistical analysis was performed by SPSS version 22.0 (IBM Corp., Armonk, NY, USA). Descriptive data were presented as the mean \pm standard deviation. Chi-square test and Fisher exact test were used for comparisons between the two groups. Risk factors

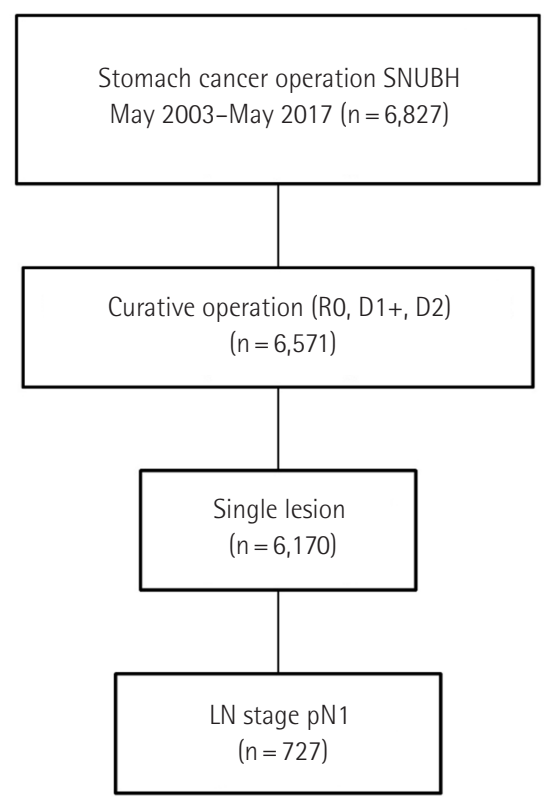

Fig. 1. Consort flow diagram. SNUBH, Seoul National University Bundang Hospital; LN, lymph node. that independently influenced skip or transversal metastases were determined by logistic regression analysis.

\section{RESULTS}

The characteristics of the patient groups are shown in Table 1. Four hundred seventy-seven (65.5\%) patients were male. Distal gastrectomy $(76.5 \%)$ and total gastrectomy $(17.2 \%)$ were the most common. The longitudinal location of tumor was greatest in the lower third region (59.0\%), followed by middle third region (22.1\%), and upper third region (18.8\%). Tumor's circumferential location was most common in the lesser curvature (42.9\%), followed by posterior wall (20.9\%), anterior wall (18.6\%), and greater curvature (14.0\%). Histologically, most cases were diagnosed as well differen-

Table 1. Clinicopathological data

\begin{tabular}{|c|c|}
\hline Characteristic & Value \\
\hline Sex (male/female) & $477 / 250$ \\
\hline Age (yr) & $60.8 \pm 12.9$ \\
\hline \multicolumn{2}{|l|}{ Surgical approach } \\
\hline DG & $556(76.5)$ \\
\hline TG & 125 (17.2) \\
\hline PG & $38(5.2)$ \\
\hline PPG & $8(1.1)$ \\
\hline \multicolumn{2}{|l|}{ LND } \\
\hline $\mathrm{D} 1+\mathrm{B}$ & $317(43.6)$ \\
\hline D2 & $410(56.4)$ \\
\hline \multicolumn{2}{|c|}{ Longitudinal location (of tumor) } \\
\hline U & 137 (18.8) \\
\hline M & $161(22.1)$ \\
\hline $\mathrm{L}$ & $429(59.0)$ \\
\hline \multicolumn{2}{|l|}{ Circular location (of tumor) } \\
\hline Lesser curvature & $312(42.9)$ \\
\hline Greater curvature & $102(14.0)$ \\
\hline Anterior wall & 135 (18.6) \\
\hline Posterior wall & 152 (20.9) \\
\hline Circular & $26(3.6)$ \\
\hline Tumor size (cm) & $4.2 \pm 2.5$ \\
\hline \multicolumn{2}{|l|}{ Histology } \\
\hline Well differentiated & $35(4.8)$ \\
\hline Moderately differentiated & $295(40.6)$ \\
\hline Poorly differentiated & $325(44.7)$ \\
\hline Poorly cohesive & $72(9.9)$ \\
\hline \multicolumn{2}{|l|}{ Lauren classification } \\
\hline Intestinal & $379(52.1)$ \\
\hline Diffuse & $294(40.4)$ \\
\hline Mixed & $54(7.4)$ \\
\hline
\end{tabular}

Values are presented as mean \pm standard deviation or number $(\%)$.

DG, distal gastrectomy; TG, total gastrectomy; $\mathrm{PG}$, proximal gastrectomy; PPG, pylorus preserving gastrectomy; LND, lymph node dissection; $U$, upper third; $M$, middle third; $L$, lower third. 
Table 2. LN station of skip metastasis according to tumor location

\begin{tabular}{lcccc}
\hline & U & M & L & Total \\
\hline LN \#7 & $9(90.0)$ & $8(61.5)$ & $17(40.5)$ & $34(52.3)$ \\
LN \#8 & - & $1(7.7)$ & $12(28.6)$ & $13(20.0)$ \\
LN \#9 & $1(10.0)$ & $1(7.7)$ & $4(9.5)$ & $6(9.2)$ \\
LN \#11p & - & $3(23.1)$ & $4(9.5)$ & $7(10.8)$ \\
LN \#12a & - & - & $3(7.1)$ & $3(4.6)$ \\
LN \#13 & - & - & $1(2.4)$ & $1(1.5)$ \\
LN \#16 & - & - & $1(2.4)$ & $1(1.5)$ \\
Total & 10 & 13 & 42 & 65 \\
\hline
\end{tabular}

Values are presented as number $(\%)$.

LN, lymph node; U, upper third; $M$, middle third; L, lower third. tiated $(4.8 \%)$, moderately differentiated (40.6\%), poorly differentiated $(44.7 \%)$, or poorly cohesive $(9.9 \%)$, which were relatively rare. Single node metastasis was found in 470 patients (64.6\%), and twonode metastases were found in 257 patients (35.4\%).

The LN station with the most skip metastasis was LN \#7 (Table 2). Especially, as the longitudinal location of the tumor was higher, skip metastasis was more likely to be LN \#7. The next most common metastatic site was LN \#8, followed by \#9, \#11p, and \#12a.

In the univariate and multivariate analyses, size of tumor was significantly correlated with skip metastasis (Table 3). The skip metastasis rate was significantly lower when tumor size was more than $4 \mathrm{~cm}$ or the circumferential location of the tumor was in the

Table 3. Factors related to the occurrence of skip metastasis

\begin{tabular}{|c|c|c|c|c|}
\hline Variable & Skip metastasis & P-value & P-value ${ }^{b)}$ & OR (95\% Cl) \\
\hline \multicolumn{5}{|l|}{ Age (yr) } \\
\hline$\leq 60$ & 30/323 (9.3) & 0.569 & 0.968 & $0.988(0.561-1.742)$ \\
\hline$>60$ & $31 / 404$ (7.7) & & & \\
\hline \multicolumn{5}{|l|}{ Sex } \\
\hline Male & 39/477 (8.2) & 0.779 & 0.669 & $1.123(0.624-1.181)$ \\
\hline Female & 22/250 (8.8) & & & \\
\hline \multicolumn{5}{|l|}{ Tstage } \\
\hline $\mathrm{EGC}(<\mathrm{T} 2)$ & $35 / 315$ (11.1) & 0.022 & 0.159 & 0.654 (0.363-1.181) \\
\hline $\mathrm{AGC}(\geq \mathrm{T} 2)$ & 26/412 (6.3) & & & \\
\hline \multicolumn{5}{|c|}{ Tubular location } \\
\hline$U$ & 10/137 (7.3) & 0.739 & & \\
\hline M & 12/161 (7.5) & & 0.745 & $0.858(0.341-2.160)$ \\
\hline L & $39 / 429(9.1)$ & & 0.791 & 1.109 (0.515-2.390) \\
\hline \multicolumn{5}{|c|}{ Circular location } \\
\hline LC & $32 / 312(10.3)$ & 0.181 & 0.015 & $0.219(0.065-0.743)$ \\
\hline $\mathrm{GC}$ & $3 / 102$ (2.9) & & & \\
\hline AW & 11/135 (8.1) & & 0.311 & $0.679(0.321-1.436)$ \\
\hline PW & 14/152 (9.2) & & 0.517 & 0.799 (0.405-1.576) \\
\hline Circular & 1/26 (3.8) & & 0.457 & $0.452(0.056-3.665)$ \\
\hline \multicolumn{5}{|l|}{ Size $(\mathrm{cm})$} \\
\hline$\leq 4$ & $46 / 414$ (11.1) & 0.003 & & \\
\hline$>4$ & 15/313 (4.8) & & 0.011 & $0.431(0.225-0.827)$ \\
\hline \multicolumn{5}{|l|}{ Histology } \\
\hline WD & 2/35 (5.7) & 0.236 & & \\
\hline MD & 23/295 (7.8) & & 0.646 & $1.425(0.315-6.449)$ \\
\hline PD & 27/325 (8.3) & & 0.323 & $2.250(0.451-11.223)$ \\
\hline$P C$ & $9 / 72(12.5)$ & & 0.146 & 3.621 (0.638-20.538) \\
\hline \multicolumn{5}{|c|}{ Lauren classification } \\
\hline Intestinal & $31 / 379$ (8.2) & 0.800 & & \\
\hline Diffuse & 24/294 (8.2) & & 0.495 & $0.736(0.305-1.774)$ \\
\hline Mixed & 6/54 (11.1) & & 0.744 & $1.195(0.410-3.487)$ \\
\hline
\end{tabular}

Values are presented as number/number (\%).

$\mathrm{OR}$, odds ratio; $\mathrm{Cl}$, confidence interval; $E G C$, early gastric cancer; $A G C$, advanced gastric cancer; $U$, upper third; $M$, middle third; $L$, lower third; LC, lesser curvature; GC, greater curvature; $A W$, anterior wall; $P W$, posterior wall; WD, well differentiated; $M D$, moderately differentiated; $P D$, poorly differentiated; $P C$, poorly cohesive.

a)Univariable. ${ }^{b)}$ Multivariable. 
Table 4. Factors related to the occurrence of transverse metastasis

\begin{tabular}{|c|c|c|c|c|}
\hline Variable & Transversal meta & P-value ${ }^{a)}$ & P-value ${ }^{\text {b) }}$ & OR $(95 \% \mathrm{Cl})$ \\
\hline \multicolumn{5}{|l|}{ Age (yr) } \\
\hline$\leq 60$ & 23/177 (13.0) & 0.272 & 0.160 & 1.535 (0.844-2.791) \\
\hline$>60$ & 41/236 (17.4) & & & \\
\hline \multicolumn{5}{|l|}{ Sex } \\
\hline Male & 44/264 (16.7) & 0.400 & & \\
\hline Female & 20/161 (16.1) & & 0.128 & 0.627 (0.344-1.144) \\
\hline \multicolumn{5}{|l|}{ T stage } \\
\hline $\mathrm{EGC}(<\mathrm{T} 2)$ & $38 / 252(15.1)$ & 0.891 & & \\
\hline$A G C(\geq T 2)$ & 26/161 (16.1) & & 0.710 & 0.908 (0.488-1.689) \\
\hline \multicolumn{5}{|c|}{ Tubular location } \\
\hline$U$ & $4 / 77$ (5.2) & 0.008 & & \\
\hline M & $11 / 85$ (12.9) & & 0.064 & $3.168(0.935-10.735)$ \\
\hline L & 49/251 (19.5) & & 0.002 & 5.709 (1.909-17.070) \\
\hline \multicolumn{5}{|c|}{ Circular location } \\
\hline LC & $51 / 311(16.4)$ & 0.433 & & \\
\hline $\mathrm{GC}$ & 13/102 (12.7) & & 0.208 & $0.645(0.326-1.277)$ \\
\hline \multicolumn{5}{|l|}{ Size $(\mathrm{cm})$} \\
\hline$\leq 4$ & 28/235 (11.9) & 0.028 & & \\
\hline$>4$ & $36 / 178(20.2)$ & & 0.035 & $1.917(1.046-3.514)$ \\
\hline \multicolumn{5}{|l|}{ Histology } \\
\hline WD & 1/18 (5.6) & 0.057 & & \\
\hline MD & 25/189 (13.2) & & 0.389 & $2.518(0.308-20.570)$ \\
\hline PD & $30 / 170(17.6)$ & & 0.203 & $4.158(0.463-37.330)$ \\
\hline$P C$ & 8/36 (8.7) & & 0.092 & $7.500(0.720-80.216)$ \\
\hline \multicolumn{5}{|c|}{ Lauren classification } \\
\hline Intestinal & $32 / 236$ (13.6) & 0.360 & & \\
\hline Diffuse & 28/148 (18.9) & & 0.883 & $1.072(0.626-2.709)$ \\
\hline Mixed & 4/29 (13.8) & & 0.652 & $0.746(0.209-2.660)$ \\
\hline
\end{tabular}

Values are presented as number/number (\%).

$O R$, odds ratio; $\mathrm{Cl}$, confidence interval; $E G C$, early gastric cancer; $A G C$, advanced gastric cancer; $U$, upper third; $M$, middle third; $L$, lower third; $L C$, lesser curvature; GC, greater curvature; WD, well differentiated; MD, moderately differentiated; PD, poorly differentiated; PC, poorly cohesive.

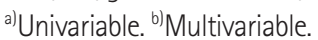

greater curvature. On the other hand, both longitudinal locations and size of tumor were associated with transversal metastases (Table 4). Transversal metastasis was significantly higher when the tumor position was located in the lower third region, or when the tumor size was greater than $4 \mathrm{~cm}$.

Information of the T classification of enrolled patients and the number of LN metastasis is in Supplementary Tables S1 and S2.

\section{DISCUSSION}

The results of the present study suggest that atypical LN metastasis may not be particularly more prevalent in AGC compared with EGC. The results presented in this study are similar with those of previous reports $[14,15]$. Specifically, there was a decrease in skip metastasis and an increase in transversal metastasis in large tumors (size $>4 \mathrm{~cm}$ ).

One of the greatest stumbling blocks in the application of SNNS is atypical metastasis, which skips the sentinel nodes. Low rate of atypical LN metastasis means cancer spreads in order from near to far, step by step. Then it may be more feasible to apply SNNS to AGC, since skip metastases are significantly decreased in AGC, as shown in this study. More studies are needed for the application of SNNS in AGC.

The most common sites at which skip metastases were observed were LNs \#7 and \#8. This is consistent with the findings of earlier studies [10,12,15-17]. This suggests that perigastric lymphatic flow may be directly connected to $\mathrm{LN} \# 7$ and $\# 8$. LN \#7 and \#8 are classified as extraperigastric LNs, but they are close to the perigastric 
$\mathrm{LN}$; therefore, it is reasonable to consider including $\mathrm{LN} \# 8$ in D1+ dissection, as well as LN \#7 in D1 dissection.

There are several hypotheses explaining the occurrence of atypical metastasis. The first is an abnormal lymphatic flow. When sentinel node biopsy is performed, it is reported that the sentinel LN may be found in the extraperigastric region and nodal metastasis can be observed in those skipping sentinel LNs [6]. There is also a view that lymphatic flow around the posterior gastric artery may be a route for skip metastasis [18]. Skip metastases being common at LNs \#7 and \#8 suggests that atypical lymphatic flow leading to those $\mathrm{LN}$ stations may be frequent. It was a reasonable decision to include node \#7, which had a high frequency of skip metastases, as part of D1 dissection in the Japanese Gastric Cancer treatment guidelines [19]. It is more like a perigastric LN than an extraperigastric LN.

The second hypothesis is that a host-related immunological defense mechanism is likely to result in skip metastasis due to the disappearance of primary sentinel LN tumor cells. This mechanism has been suggested because the presence of micrometastasis in EGC does not affect the prognosis [20].

Other possibilities may be that cancer cells bypass the sentinel LN and metastasize to the remote LN stations [14].

In this study, we limited the number of metastatic LNs to one or two. If positive metastatic LNs are too many, it is difficult to predict the lymphatic flow. To clarify the LN station in which the first or second atypical metastasis occurred, the target tumor stage of this study was limited to the $\mathrm{N} 1$ stage.

The skip metastasis rate was significantly lower when the tumor was located circumferentially in the greater curvature. This is probably because the distance between the metastatic LN and the tumor is much longer when the tumor location is in the greater curvature compared with cases where the tumor location is the lesser curvature.

The present study has some limitations. Despite the large data, it is obtained from a single institution. Secondly, due to the retrospective nature of this study, some bias of sources may be inevitable.

In conclusion, large tumors and tumors located in the lower third region were more likely to have transversal metastases than others, but skip metastasis was observed less frequently in large tumors. Tumor depth was not associated with atypical metastasis. Caution should be taken when performing SNNS for tumors located in the lower part of the stomach, or for large tumors, but SNNS might be considered in AGC as well as in EGC.

\section{CONFLICT OF INTEREST}

No potential conflict of interest relevant to this article was reported.

\section{SUPPLEMENTARY MATERIALS}

Supplementary Table S1. The numbers of positive lymph node according to $T$ stage in gastric cancer

Supplementary data can be found: https://doi.org/10.14216/ kjco.19018.S1

Supplementary Table S2. The portions of N stage according to tumor advance

Supplementary data can be found: https://doi.org/10.14216/ kjco.19018.S2

\section{REFERENCES}

1. Jo MJ, Park JY, Song JS, Kook MC, Ryu KW, Cho SJ, et al. Biopathologic features and clinical significance of micrometatasis in the lymph node of early gastric cancer. World J Gastroenterol 2015;21: 667-74.

2. Guideline Committee of the Korean Gastric Cancer Association (KGCA), Development Working Group \& Review Panel. Korean practice guideline for gastric cancer 2018: an evidence-based, multi-disciplinary approach. J Gastric Cancer 2019;19:1-48.

3. Japanese Gastric Cancer Association. Japanese gastric cancer treatment guidelines 2014 (ver. 4). Gastric Cancer 2017;20:1-19.

4. Kim DW, Jeong B, Shin IH, Kang U, Lee Y, Park YS, et al. Sentinel node navigation surgery using near-infrared indocyanine green fluorescence in early gastric cancer. Surg Endosc 2019;33:1235-43.

5. Kitagawa Y, Takeuchi H, Takagi Y, Natsugoe S, Terashima M, Murakami N, et al. Sentinel node mapping for gastric cancer: a prospective multicenter trial in Japan. J Clin Oncol 2013;31:370410.

6. Ichikura T, Sugasawa H, Sakamoto N, Yaguchi Y, Tsujimoto H, Ono S. Limited gastrectomy with dissection of sentinel node stations for early gastric cancer with negative sentinel node biopsy. Ann Surg 2009;249:942-7.

7. Bravo Neto GP, Dos Santos EG, Victer FC, Neves MS, Pinto MF, Carvalho CE. Sentinel lymph node navigation surgery for early gastric cancer: is it a safe procedure in countries with non-endemic gastric cancer levels? A preliminary experience. J Gastric Cancer 2016;16:14-20.

8. Park DI, Park YS, Son SY, Lee JH, Lee HS, Park YS, et al. Long-term oncologic outcomes of laparoscopic sentinel node navigation surgery in early gastric cancer: a single-center, single-arm, phase II trial. Ann Surg Oncol 2018;25:2357-65.

9. Kosaka T, Ueshige N, Sugaya J, Nakano Y, Akiyama T, Tomita F, et al. Lymphatic routes of the stomach demonstrated by gastric carcinomas with solitary lymph node metastasis. Surg Today 1999;29: 695-700. 
10. Lee SE, Lee JH, Ryu KW, Cho SJ, Lee JY, Kim CG, et al. Sentinel node mapping and skip metastases in patients with early gastric cancer. Ann Surg Oncol 2009;16:603-8.

11. Kim DH, Choi MG, Noh JH, Sohn TS, Bae JM, Kim S. Clinical significance of skip lymph node metastasis in gastric cancer patients. Eur J Surg Oncol 2015;41:339-45.

12. Lee JH, Lee HJ, Kong SH, Park DJ, Lee HS, Kim WH, et al. Analysis of the lymphatic stream to predict sentinel nodes in gastric cancer patients. Ann Surg Oncol 2014;21:1090-8.

13. Huang B, Wang Z, Sun Z, Zhao B, Xu H. A novel insight of sentinel lymph node concept based on 1-3 positive nodes in patients with pT1-2 gastric cancer. BMC Cancer 2011;11:18.

14. Choi YY, An JY, Guner A, Kang DR, Cho I, Kwon IG, et al. Skip lymph node metastasis in gastric cancer: is it skipping or skipped? Gastric Cancer 2016;19:206-15.

15. Park SS, Ryu JS, Min BW, Kim WB, Kim SJ, Kim CS, et al. Impact of skip metastasis in gastric cancer. ANZ J Surg 2005;75:645-9.

16. Li C, Kim S, Lai JF, Oh SJ, Hyung WJ, Choi WH, et al. Solitary lymph node metastasis in gastric cancer. J Gastrointest Surg 2008;12:550-4.

17. Miwa K, Kinami S, Taniguchi K, Fushida S, Fujimura T, Nonomura A. Mapping sentinel nodes in patients with early-stage gastric carcinoma. Br J Surg 2003;90:178-82.

18. Ishii K, Ishida M, Sugisawa N, Murakami M, Ono T, Tachibana T, et al. Lymph nodes around the posterior gastric artery: their existence, frequency, and clinical implications. Surg Today 2018;48: 916-20.

19. Japanese Gastric Cancer Association. Japanese gastric cancer treatment guidelines 2010 (ver. 3). Gastric Cancer 2011;14:113-23.

20. Morgagni P, Saragoni L, Scarpi E, Zattini PS, Zaccaroni A, Morgag$\mathrm{ni}$ D, et al. Lymph node micrometastases in early gastric cancer and their impact on prognosis. World J Surg 2003;27:558-61. 
Supplementary Table S1. The numbers of positive LN according to T stage in gastric cancer

\begin{tabular}{|c|c|c|c|c|c|c|c|}
\hline \multirow{2}{*}{ No. of positive LNs } & \multicolumn{6}{|c|}{ T stage } & \multirow{2}{*}{ Total } \\
\hline & T1a & $\mathrm{T} 1 \mathrm{~b}$ & T2 & T3 & T4a & $\mathrm{T} 4 \mathrm{~b}$ & \\
\hline 0 & 2,013 & 1,298 & 389 & 243 & 63 & 9 & 4,015 \\
\hline 1 & 42 & 185 & 99 & 91 & 40 & 13 & 470 \\
\hline 2 & 22 & 66 & 52 & 75 & 35 & 7 & 257 \\
\hline 3 & 8 & 47 & 33 & 68 & 37 & 7 & 200 \\
\hline 4 & 4 & 23 & 23 & 57 & 33 & 5 & 145 \\
\hline 5 & 8 & 12 & 24 & 38 & 31 & 6 & 119 \\
\hline 6 & 2 & 7 & 14 & 41 & 20 & 7 & 91 \\
\hline 7 & 3 & 16 & 15 & 28 & 28 & 7 & 97 \\
\hline 8 & 1 & 1 & 8 & 29 & 28 & 4 & 71 \\
\hline 9 & 0 & 7 & 9 & 24 & 19 & 4 & 63 \\
\hline 10 & 0 & 6 & 5 & 20 & 14 & 2 & 47 \\
\hline 11 & 0 & 1 & 4 & 26 & 22 & 3 & 56 \\
\hline 12 & 1 & 2 & 0 & 15 & 18 & 2 & 38 \\
\hline 13 & 0 & 0 & 2 & 14 & 8 & 5 & 29 \\
\hline 14 & 0 & 1 & 3 & 6 & 18 & 4 & 32 \\
\hline 15 & 0 & 1 & 1 & 12 & 10 & 2 & 26 \\
\hline 16 & 0 & 4 & 1 & 13 & 13 & 2 & 33 \\
\hline 17 & 0 & 0 & 0 & 7 & 16 & 3 & 26 \\
\hline 18 & 0 & 1 & 3 & 8 & 9 & 3 & 24 \\
\hline 19 & 0 & 0 & 0 & 4 & 14 & 1 & 19 \\
\hline 20 & 0 & 0 & 3 & 5 & 12 & 2 & 22 \\
\hline 21 & 0 & 0 & 0 & 6 & 11 & 3 & 20 \\
\hline 22 & 0 & 1 & 3 & 9 & 7 & 2 & 22 \\
\hline 23 & 0 & 0 & 1 & 5 & 17 & 1 & 24 \\
\hline 24 & 0 & 1 & 1 & 4 & 6 & 2 & 14 \\
\hline 25 & 0 & 1 & 1 & 0 & 14 & 2 & 18 \\
\hline 26 & 0 & 0 & 0 & 2 & 7 & 3 & 12 \\
\hline 27 & 0 & 0 & 0 & 2 & 9 & 3 & 14 \\
\hline 28 & 0 & 0 & 0 & 1 & 11 & 3 & 15 \\
\hline 29 & 0 & 1 & 0 & 4 & 5 & 0 & 10 \\
\hline 30 & 0 & 1 & 0 & 3 & 4 & 0 & 8 \\
\hline 31 & 0 & 1 & 0 & 1 & 3 & 0 & 5 \\
\hline 32 & 0 & 0 & 0 & 0 & 3 & 1 & 4 \\
\hline 33 & 0 & 0 & 1 & 1 & 5 & 1 & 8 \\
\hline 34 & 0 & 0 & 1 & 1 & 7 & 0 & 9 \\
\hline 35 & 0 & 0 & 0 & 3 & 5 & 0 & 8 \\
\hline 36 & 0 & 0 & 2 & 0 & 5 & 0 & 7 \\
\hline 37 & 0 & 0 & 0 & 1 & 6 & 1 & 8 \\
\hline 38 & 0 & 0 & 1 & 1 & 7 & 0 & 9 \\
\hline 39 & 0 & 0 & 0 & 0 & 2 & 0 & 2 \\
\hline 40 & 0 & 0 & 0 & 0 & 4 & 1 & 5 \\
\hline 41 & 0 & 0 & 0 & 4 & 1 & 0 & 5 \\
\hline 42 & 0 & 0 & 0 & 0 & 4 & 0 & 4 \\
\hline 43 & 0 & 0 & 1 & 1 & 3 & 1 & 6 \\
\hline 44 & 0 & 0 & 1 & 0 & 2 & 0 & 3 \\
\hline 45 & 0 & 0 & 0 & 0 & 6 & 1 & 7 \\
\hline 46 & 0 & 0 & 0 & 0 & 3 & 0 & 3 \\
\hline 47 & 0 & 0 & 0 & 0 & 2 & 0 & 2 \\
\hline 48 & 0 & 0 & 0 & 0 & 4 & 0 & 4 \\
\hline 49 & 0 & 0 & 0 & 0 & 1 & 0 & 1 \\
\hline 50 & 0 & 0 & 0 & 1 & 2 & 0 & 3 \\
\hline 51 & 0 & 0 & 0 & 1 & 3 & 0 & 4 \\
\hline 54 & 0 & 0 & 1 & 0 & 1 & 0 & 2 \\
\hline 55 & 0 & 0 & 0 & 0 & 1 & 0 & 1 \\
\hline 56 & 0 & 0 & 0 & 1 & 1 & 0 & 2 \\
\hline 57 & 0 & 0 & 0 & 0 & 1 & 0 & 1 \\
\hline 58 & 0 & 1 & 0 & 0 & 1 & 0 & 2 \\
\hline 60 & 0 & 0 & 0 & 0 & 2 & 0 & 2 \\
\hline 62 & 0 & 0 & 0 & 0 & 2 & 0 & 2 \\
\hline 63 & 0 & 0 & 0 & 1 & 0 & 0 & 1 \\
\hline 64 & 0 & 0 & 0 & 2 & 1 & 0 & 3 \\
\hline 65 & 0 & 0 & 0 & 0 & 1 & 0 & 1 \\
\hline 68 & 0 & 0 & 0 & 1 & 0 & 0 & 1 \\
\hline 70 & 0 & 0 & 0 & 0 & 1 & 1 & 2 \\
\hline 85 & 0 & 0 & 0 & 0 & 1 & 0 & 1 \\
\hline 87 & 0 & 0 & 0 & 0 & 0 & 1 & 1 \\
\hline 89 & 0 & 0 & 0 & 0 & 1 & 0 & 1 \\
\hline 92 & 0 & 0 & 0 & 0 & 1 & 0 & 1 \\
\hline 102 & 0 & 0 & 0 & 0 & 1 & 0 & 1 \\
\hline 104 & 0 & 0 & 0 & 0 & 1 & 0 & 1 \\
\hline Total no. (\%) & $2,104(34.1)$ & $1,685(27.3)$ & 702 (11.4) & 880 (14.3) & 674 (10.9) & $125(2.0)$ & $6,170(100.0)$ \\
\hline
\end{tabular}

LN, lymph node. 
Supplementary Table S2. The portions of N stage according to tumor advance

\begin{tabular}{lccc}
\hline & EGC & AGC & Total \\
\hline N0 & $3,311(87.4)$ & $704(29.6)$ & $4,015(65.1)$ \\
N1 $^{\text {a) }}$ & $315(8.3)$ & $412(17.3)$ & $727(11.8)$ \\
N2 & $111(2.9)$ & $444(18.6)$ & $555(90)$ \\
N3a & $40(1.1)$ & $419(17.6)$ & $459(7.4)$ \\
N3b & $12(0.3)$ & $402(16.9)$ & $414(6.7)$ \\
Total & $3,789(61.4)$ & $2,381(38.6)$ & $6,170(100.0)$ \\
\hline
\end{tabular}

Values are presented as number (\%).

EGC, early gastric cancer; AGC, advanced gastric cancer.

${ }^{a}$ Only $\mathrm{N} 1$ gastric patients was analyzed in this study. 